\title{
Mobile Interaction Design: Techniques for Early Stage In-Situ Design
}

\author{
Marco de Sá, Luís Carriço \& Carlos Duarte \\ LaSIGE and Faculty of Sciences, University of Lisbon \\ Portugal
}

\section{Introduction}

The recent globalization of mobile technology and its overwhelming presence on everyday life through various societal groups and activities has raised its importance to unprecedented levels. Mobile devices' diverse shapes, small size and distinctive characteristics impel their use in diverse and ubiquitous scenarios, cementing their presence within our work, social and entertainment activities. Accordingly, as they assume a greater meaning and a wider role of functionalities, a corresponding amount of new usage paradigms is also emerging. Consequentially, designers are increasingly faced with new design challenges, needing to cope with added difficulties of creating solutions for multiple contexts, users, purposes and new ubiquitous usage behaviours. Simultaneously, they need to cope with and leverage the small size factor and the peculiar or mixed interaction modalities (e.g., touch screen in concert with keyboard or voice) that define the trends of emerging mobile devices.

Contrastingly, design problems for mobile devices, and corresponding solutions, have only recently begun to be partially and superficially addressed. Difficulties and challenges are spread through various stages of design. Three phases are particularly interesting: (1) requirements and data gathering on mobile contexts; (2) prototyping for small devices and (3) evaluation on real-world settings. Currently used approaches and existing methodologies still lack specific techniques to support design on such demanding conditions, hindering the design process and resulting in poor software regarding usability. Even recent approaches generally rely on simulations, lab experiences or derive directly from non-mobile techniques, colliding with studies that have clearly demonstrated the need to take the design process out of the lab when it comes to mobile devices.

This book chapter focuses on these problems and discusses recent advances on mobile interaction design, reviewing existing attempts to overcome the added challenges brought by mobility, pervasiveness and mobile devices' characteristics. As its main contribution, it identifies key concerns and issues brought by mobility, also presenting ways to complement current efforts and proposing new approaches that aim at overcoming existing challenges and problems. It introduces findings and work developed thus far, offering improvements and solutions that tackle out-of-the-lab design procedures and support in-situ participatory design and evaluation. These approaches are compiled within a User Centred Design (UCD) methodology that emphasizes initial stages of design and identifies techniques and 
guidelines for (1) early stage data gathering and scenario generation for design; (2) lowfidelity prototyping techniques particularly suited to mobile devices which also propel an easier transition to (3) evaluation on real-world contexts and settings. Case studies where these techniques have been applied are presented, detailing the used procedures and tools, achieved results and eliciting the implications and benefits that emerged from the application of such techniques. As a conclusion, suggestions and guidelines on how to apply similar endeavours on different domains are presented, also defining further research goals and directions within mobile interaction design.

\section{Motivation and Related Work}

The somewhat recent appearance of ubiquitous and pervasive computing, supported by an ever growing diversity of mobile technology, has introduced a set of additional challenges into the design process of interactive application for mobile devices (Blom et al., 2005). While attempting to overcome such difficulties, designers often choose to port to mobile devices, existing applications available for larger platforms, adjusting the necessary details (Nakajima, 2006). However, such approach generally leads to cumbersome and unusable applications that even if containing brilliant content, quickly become obsolete and avoided by users (Brewster, 2002; Lee, 2003).

A major factor for designers to adopt such strategies is the absence of specific methodologies for mobile, handheld and ubiquitous devices (Hagen, 2005; Raptis, 2005). Although some guidelines, available in current user-centred design (UCD) methodologies, might apply to this specific design process, the unique features and constraints that ubiquity, pervasiveness and the devices' physical characteristics introduce require new, or at least deeply refined, approaches.

Three design stages are particularly sensitive and present a wider set of difficulties (Sá \& Carriço, 2006a):

- (1) Gathering requirements in mobile scenarios, where the constant use of the mobile device or application is done in changing contexts, introduces details that are hardly detectable with the use of traditional methods. One can imagine, for instance, that if all contexts are known, traditional methods can acceptably assess the influence of each context in specific scenarios. However, the implications of changing from one context to another and how this might influence the user and his/her requirements towards the applications fail to be understood.

- (2) On the sketching process and construction of low-fidelity prototypes, several problems that retract from the process are also evident. Low-fidelity prototypes are, as currently used, poorly suited to the peculiarities of mobile devices and their usage scenarios (Sá \& Carriço, 2006a). These ill-suited prototyping methods also have implications on the later stages of evaluation.

- (3) In fact, when it comes to the evaluation of mobile applications, given the absence of specific methods and guidelines, this stage is frequently discarded (Kjeldskov \& Graham, 2003). Overall, the difficulties inherent to the design process of applications for such devices urge for different approaches and extensions to current user-centred design processes.

Aligned with these preoccupations, mobile interaction design has been recently addressed on various emerging researches (Hagen et al., 2005; Jones \& Marsden, 2006; Kjeldskov and Stage, 2003; Weiss, 2002). Generally, these approaches try to adjust existing and traditional techniques to mobile settings or, in some cases, introduce new methods that cope with ubiquity and the small size factor that commonly characterizes mobile devices. Nevertheless, serious deficiencies are pointed in the available literature affecting the 
advance of mobile interaction design (Hagen et al, 2005; Kjeldskov \& Graham, 2003; Lee, 2003; Sá \& Carriço, 2006a; Marcus \& Chen, 2002). As a consequence, it is common practice to overlook determinant design stages such as prototyping and evaluation (Kjeldskov \& Stage, 2003; Nielsen et al., 2006), which impacts directly on the quality of available applications (Lee, 2003). Alternatively, simulation and role playing are recurrently used as quick patches (Barnard et al., 2005; Svanaes \& Seland, 2004). However, these also fall short while trying to grasp realistic usage requirements (Nielsen et al., 2006).

The main motivation behind this work, builds upon the aforementioned lack of relevant guidance within mobile interaction design and on the difficulties that emerged during the design of several projects for mobile devices (Beyer \& Holtzblatt, 1998; Mayhew, 1999). Overall, problems affect design teams throughout the entire process, requiring constant adjustments to each stage particularly hindering, as mentioned, the analysis and requirements gathering, prototyping and evaluation phases. Each of the following sections stresses, in deeper detail, the particular issues that affect the aforementioned design stages addressing the state-of-the-art and existing approaches to each task and design stage.

\subsection{Data Gathering}

Data gathering is the essential bootstrap to the design process of most interactive applications. This stage's goal is to provide designers with relevant data on how users work or act within their usual working settings and how a technological solution can improve that process. Several techniques can be used to achieve this purpose. The most common are questionnaires, interviews, contextual inquiry and user observation.

User questionnaires are, as the name points, questionnaires that are delivered to large groups of users trying to gather opinions and data based directly on the users' input and perspective on existing issues. The remaining techniques aim at gathering richer information and data by including designers directly on the process and by introducing interaction between designers and future users, allowing the former to make use of their expertise and experience while gathering information from users.

Interviews are used to obtain personal opinions from representative users from a target audience, gathering information on needs, wishes and preferences. Interviews are generally preceded by the construction of a script and questionnaire that is presented and responded orally. Although interviews can provide richer data and information when compared to questionnaires, mainly because designers can adjust, on-the-fly, their questions as the interview goes along, as well as to witness users' reactions to specific questions and details, they are generally applied to a much smaller population. Given the time and resources that they require, it is difficult to interview as many target users as those that can be reached through questionnaires (e.g., on-line questionnaire or street survey). Furthermore, interviews can be biased and influenced by the interviewer's input (Read et al., 2004). However, a major advantage is that they can be used with a varied type of population (e.g., children, elderly, visually impaired, etc) (Consolvo et al., 2004). Still, they are very focused on user input and might provide little information regarding paramount details (e.g., location, settings, and cooperation) that result from activities that users accomplish even without noticing. To overcome some of these problems designers often conduct interviews in context while observing some of the user's activities. This type of dynamic and on-thespot interview is called contextual inquiry. With contextual inquiry the interview is conducted on the location where users work, with the usual settings, focusing activities that are taking place during that interview. It usually includes conversation and interaction 
between the designer that conducts the interview and the user that is accomplishing the activities.

Since it occurs at the location where work is usually accomplished, it provides extra detail on the work context and existing problems. It merges some of the advantages of interviewing with some of the advantages of user observation. It has also been successfully used on critical scenarios. Coble et al. used the contextual inquiry method to assess requirements on a clinical setting, overcoming previous experiences that provided poor information on physicians' needs and consequently ended with poorly designed solutions and attempts (Coble et al., 1997).

However, taking into consideration ubiquitous applications, where tasks can be accomplished on multiple settings and extend through various contexts, contextual inquiry becomes extremely difficult, if not impossible to apply. Conducting contextual inquiry on a ubiquitous activity would necessary imply that the interviewer would have to follow the user around interrupting him whenever necessary in order to ask questions. Moreover, user's behaviour is usually affected by designer's questions and interruptions, thus providing a less realistic notion of how activities are accomplished, users' reactions and if, how and why behaviour changes occur. To avoid this type of problem, a simpler method is direct user observation. The user observation technique comprehends direct observation of a user accomplishing specific activities on realistic settings without any interference by the designer that is observing. Its main advantages reside on the richness of the gathered data and the unbiased perspective of the work flow. However, in order to correctly observe users while accomplishing specific task some cautions have to be taken. For instance, in Mazzone et al. (2004), the authors conducted a preliminary ethnographic study in order to understand the best locations and settings and where and how to observe the users. Nevertheless, similarly to contextual inquiry, user observation has limitations when ubiquity and mobility are involved. To properly observe a user while using a mobile device, the designer would have to follow the user everywhere while accomplishing a specific task. Moreover, the designer would have to be extremely close to the user in order to view the user's interaction with the device possibly hindering the process which also poses restrictions to the user's behaviour, especially during private tasks.

Throughout various experiences, on mobile design, the application of these techniques, suggested by the used UCD methodologies, posed problems and proved to be inadequate to some of the settings and activities that were being addressed. On pervasive and mobile activities as those that were targeted on the projects at hands (e.g., thought registration and activity scheduling during user's daily lives, Cognitive Behavioural Therapy, homework), these data gathering techniques failed to provide sufficient detail and reliable data for designers to use (Sá \& Carriço, 2006). Globally, these difficulties pointed the need for adjusted and more flexible means and techniques do gather data and analyse requirements on mobile and ubiquitous settings, especially for pervasive activities.

\subsection{Prototyping}

Prototypes can be either low-fidelity prototypes, focused on this chapter, used in early stages of design, or highly usable prototypes, close to the final application. During the design process of a certain software program, prototypes allow designers to test their ideas and concepts with final users before the final product is completed (Beyer \& Holtzblatt, 1998; Hanington, 2006; Mayhew, 1999). Low-fidelity (low-fi) prototypes are non-functional 
User Interfaces (UIs) sketched on paper cards or post-its, put together with glue, pins and cut out with scissors (Beyer \& Holtzblatt, 1998; Holtzblatt et al., 2005; Robert A. Virzi, 1996), that are used to simulate an actual system, while evaluating its features and detecting its flaws during early design stages. Prototypes are an essential tool for UCD and many other methodologies.

In (Frishberg, 2006a) the importance of low-fidelity prototypes to drive design and evaluate ideas with low-cost is addressed. The author stresses the need to provide users with objects that reflect ideas, illustrating assumptions and concepts on a physical and tangible manner. Accordingly, the use of prototypes facilitates user interaction with design concepts and challenges users to provide input and generate ideas. However, advantages for designers are also paramount. As detailed in (Rosenberg, 2006), low-fidelity prototypes provide a way for designers to assess user satisfaction at very early stages. They also provide a common reference point for all the design team members, improve the completeness of the product's functional specification and substantially reduce the total development cost of the product. Accordingly, this type of easy to use and highly configurable prototypes is particularly interesting for early design stages since they can be quickly built using inexpensive material (Chandler et al., 2002; Connelly et al., 2005; Kangas \& Kinnunen, 2005).

Summarizing, low-fidelity prototypes, present an important tool for designers to test their designs and solutions during early design phases (Beyer \& Holtzblatt, 1998; Black \& Hawkes, 2006; Mayhew, 1999; Robert A. Virzi, 1996; Svanaes \& Seland, 2004; Weiss, 2002). They enable developers with a quick and inexpensive way to evaluate and assess some design ideas without programming and implementing real and functional solutions. In fact, in some cases, their efficiency is almost as high as software prototypes. Several studies (Kangas \& Kinnunen, 2005; Robert A. Virzi, 1996) have demonstrated that paper prototypes can be efficiently used to prevent posterior design errors and unusable applications.

However, even if they are to be viewed as a mere designing tool, many times these are perceived by users as a very resembling solution to the final application. In (Holmquist, 2005), the author points designers' attention to avoid misleading users while creating prototypes. Often, designers create very appealing prototypes which please users but are too expensive or impossible to actually implement generally leading to failure or disappointment. On a more user concerned level, this can also affect the usability of the resulting applications whereas during evaluation no major usability issues are detected while on a final version they are present in a very obtrusive way. This is particularly true for mobile devices. For instance, using a Letter sized paper to draw a UI, and using post-its as menus might be an acceptable way to prototype a desktop application. However, when it comes to mobile applications, specific attention must be taken to details which compromise their usage on real settings. In fact, here, paper prototyping may pose a considerable problem and mislead users. For instance, using a post it with a few key buttons to simulate the use of a PDA with a GPS card might seem a great idea to test a future application. However, using the real device on one's hand throughout the day may be a demanding task, requiring a much different UI or might be unsuitable for certain users (e.g., elderly) or scenarios (e.g., hiking). Testing an application with an artificial keyboard might work if the size of the letters is large, but using a real device keyboard might be quite different, suggesting the need to use alternative solutions. These details may pass without notice if one does not create suitable prototypes and perform tests on real life scenarios. It is true that low-fidelity prototypes need to be quick to build and easy to use (Beyer \& Holtzblatt, 1998; 
Robert A. Virzi, 1996) but the trade-off between the effort in building them and the misleading results that they might provide needs to be carefully analysed. To be as profitable and useful as acclaimed, low-fidelity prototypes for mobile devices require special attention. In general, when ill-implemented they might even produce the opposite effect than that expected (Hanington, 2006; Holmquist, 2005) since users and sometimes designers, tend to visualize mobile prototypes, even low-fidelity ones, as extremely resembling to final solutions. If care is not taken, this misleading conduct can affect the evaluation process, the use of the prototype itself, and the final result.

Also, and of great importance, is the fact that with mobile prototypes, external characteristics to the sketches may influence the validity of the prototype. Details such as weight, interaction modalities, size or shape may influence the way in which the sketch is perceived. Furthermore, simpler restraints such as the screen resolution or area might imply that common prototyping techniques need to be adapted to such details. Traditionally, mobile prototypes are regarded as just another case of prototyping. Clearly, due to the aforementioned characteristics, special attention must be taken when prototyping for mobile devices, suggesting the need for new approaches regarding this practice. Currently, lowfidelity prototyping for mobile devices is generally done in a classic way, creating sketches on paper cards and using post-its as buttons or menus. The few examples available in literature apply common techniques with no particular emphasis on the specific characteristics of the devices and their interaction. Recently, research directed to this stage of design, applied to mobile devices (Hanington, 2006; Holmquist, 2005; Weiss, 2005) including some of the experiences that originated this work (Sá \& Carriço, 2006) has addressed these issues. Given mobile devices' portability and adequateness to intensive usage and their physical characteristics and peculiar interaction modalities, the distinction between the device and the UI, although rarely addressed and implemented, seems to be crucial to take into account while creating mobile low-fidelity prototypes.

In (Hanington, 2006) this topic is discussed and some experiences described. The author explains how paper and physical prototyping are essential to provide users with notions such as screen real estate, input mechanisms, and weight and so on. Moreover, the authors suggest that integrating the device prototype with the sketching process inspires inventive and creative products. The research experiences that were conducted included the construction of physical prototypes using materials such as foam, slides and other found materials. Sketches were drawn on paper and used in concert with the physical prototypes. Positive results demonstrated that merging both concepts and actually creating physical prototypes enhanced the evaluation and testing processes.

Other research experiences that focus on how prototypes should be built and what should be their characteristics consider the utilization of various fidelities of prototypes, including more or less details depending on what is being evaluated. In (McCurdy et al., 2006) the authors point the differences between prototype fidelities and present an example of a mixed-fidelity prototype. Accordingly, they suggest five different dimensions that can be adjusted according to the evaluation that needs to be conducted. The first is the visual refinement which can vary from hand drawn sketches to pixel-accurate mockups. The second dimension refers to the breadth of functionality which defines what the user can actually do with the prototype, ranging from no functionality where no action can be done with the prototype, to highly functional prototypes where most of the final functionalities are available. Closely related is the third dimension, depth of functionality, which pertains 
to how accurate the simulation and level of functionality of each feature is. Finally, the richness of interactivity and the richness of the data model which refer to how close the interaction with the prototype is to the final product and to how representative the actual domain data employed in the prototype is. Overall, these dimensions allow designers to categorize their prototypes depending on the type of UI or device they want to evaluate or design for. As previously mentioned, given mobile device's characteristics, dimensions such as the level of visual refinement and richness of functionality, especially on low-fi prototypes, are paramount.

Globally, it is clear that prototyping is an extremely positive endeavour that allows designers to propel design, include users on the design process and evaluate concepts and ideas on early stages of design. However, as some research already points, with mobile devices particular care must be taken while generating effective prototypes. Current literature, although stating some evidences of evolution and suggesting some new approaches, does not provide practical guidelines on how to develop or use low-fi prototypes for mobile devices. Existing examples, although well intentioned, offer suggestions that do not comply with the evaluation that is required on mobile usability, showing inability to cope with the specific characteristics that mobile devices and their usage requires. Other examples found in the literature follow different approaches, pointing directions which generally require expensive material and can not be easily used on early stages (Sá \& Carriço, 2008).

On the experiences that motivated this work, as projects evolved to the prototyping stage, the type of procedures that were, as abovementioned, proposed by the literature introduced a set of problems on the usage of the prototypes that were created. Components and their size generally confounded users and resulted in unrealistic, misunderstood and quickly rejected prototypes. Since screen measurements (e.g., fonts, scroll-bars) were sometimes disregarded and prototypes quickly hand drawn, the information that each card started to include was excessive and impossible to include on actual devices. User participation was problematic since users' requests, suggestions and their own sketches were unattainable given the dimensions and features of the targeted devices. Moreover, together with the design fidelity details (e.g., size, components), more realistic tools than post-its or cards, as exemplified in (Beyer \& Holtzblatt, 1998; Jones \& Marsden, 2006; Mayhew, 1999; Weiss, 2002) were needed. These materials also mislead users regarding weight, size or the interaction with the devices. The cards often deteriorate during outside evaluation sessions and simulations, especially each time users try to put a paper PDA prototype in his/her pocket, while mimicking their common behaviour on common daily life situations.

Globally, both literature and some of the problems and results from the mentioned experiences stressed the need for particular care and new prototyping methods for mobile devices, addressing issues such as shape, format, fidelity levels, adequateness to evaluation and so on.

\subsection{Usability Evaluation}

Usability evaluation out of the lab, especially during early design stages, which has been proved to be critical on various design projects (Sá et al, 2007; Reis et Al, 2008), is not yet a common procedure (Kjeldskov \& Graham, 2003). Although some researches have addressed this subject, stressing its importance and effective results (Duh et al., 2006; Jones \& Marsden, 2006; Kjeldskov \& Graham, 2003; Nielsen et al., 2006), most rely on simulations and role 
playing and generally take place within controlled scenarios (Barnard et al., 2005; Kjeldskov \& Stage, 2003; Svanaes \& Seland, 2004). Accordingly, used methodologies provide no insight on how to conduct any type of multiple context/setting evaluation.

For instance, the use of the Wizard of Oz technique (Kelley, 1984) with mobile prototypes can be extremely demanding. Following a user while he/she tests a prototype on every imagined scenario might even be impossible to achieve. The procedure itself generally causes ethical and social problems and provides erroneous results. To support this type of activity, some approaches have been experimented and technological systems (Carter et al., 2007; Froelich et al., 2007; Reich et al., 2007) are being developed to support the remote evaluation of mobile applications on real-settings. However, they generally require working software prototypes and applications or use expensive material that is not commonly accessible.

Although, from a generic perspective, traditional techniques have been successfully used on a variety of tests for different systems and applications, the particular requirements that mobile devices introduce, mainly due to their use on a multitude of contexts, requires further effort and adjustments to these methods (Hagen et al., 2005; Kjeldskov \& Stage, 2003; Nielsen et al., 2006; Scholtz \& Consolvo, 2004). Recent research experiences suggest that given their intensive and pervasive use, mobile devices and correspondent applications should be evaluated on multiple and realistic settings. Contrarily to fixed and desktop hardware, when using mobile devices, users are constantly faced with distractions and obstacles and often accomplish several tasks at the same time. Accordingly, recent studies have been addressing this topic. Duh et al. conducted a series of tests that aimed at determining the differences between usability tests conducted within laboratories or field tests with mobile devices (Duh et al., 2006). Similar tests took place in the laboratory and on the field. In the laboratory test, digital cameras were used to capture the mobile device's interface and the user's facial expressions. On the field test a camera assistant captured only the facial expression of the user while walking around a specific setting. Results revealed field observation and tests enabled the detection of many more usability problems. Furthermore, most of the problems that were detected on the field tests were categorized as critical.

Nielsen at al. conducted a similar experience (Nielsen et al., 2006). Their results also demonstrate that field tests provide more valid results and identify significantly more usability problems. Moreover, task completion times almost duplicated on field tests and on real usage settings, suggesting that laboratory tests might provide erroneous results.

In Barnard et al. the authors show, through a series of tests how context affects human performance in mobile computing systems (Barnard et al., 2007). In this study, users were requested to perform some activities on different scenarios. The experiences focused lighting and movement (e.g., if the user is standing or walking) conditions. Results show that the user's surroundings can greatly influence his/her behaviour. Accordingly, the authors suggest that context and the environment should take a primordial role while designing and evaluating mobile applications and new methods to achieve so are necessary. Even so, the reported experiences were conducted within controlled and simulated environments. Despite the evident advantages of field evaluation tests, there are still few reports of successful experiences and even fewer suggestions and guidelines on how to address such type of evaluation. Kjeldskov \& Graham presented a study that clearly demonstrates that designers tend to engineer systems using applied approaches rather than designing and 
evaluating them (Kjeldskov \& Graham, 2003). Furthermore, even when these are evaluated, evaluation sessions are generally conducted on laboratories. In addition, the authors conclude that contextual evaluation and phenomena observation on real settings is not widely used, presenting an increasingly required research track on the field of HCI. Curiously, one of these authors conducted a survey on new emerging techniques to evaluate mobile systems on laboratories. Here, the authors justify this approach, opposing to field and contextual evaluation, due to three difficulties that are generally felt while trying to do so. The first pertains to the scenarios and settings in which tests should be done. It is difficult to select specific situations that represent a set of contexts to evaluate. Summarizing, key settings are hard to identify.

The difficulty to apply existing evaluation techniques on mobile settings is the second reason whilst the third is caused by the complicated data collection. On field evaluations it is demanding to cope with the physical surroundings and environment that might affect the set-up. This is noticeable both on the designers that gather the data and on the users which accomplish tasks. On the other hand the authors defend that in laboratory settings these difficulties can be reduced. Although user mobility can be difficult to recreate, according to the authors, it is preferable to lose realism towards a better quality of collected data. Accordingly, they propose two frameworks for mobile evaluation in laboratories. The first focuses on the user's physical movements while using the mobile system and on the attention needed to navigate through the software. These two dimensions allow designers to arrange tests according to different configurations simulating various levels of movement and attention. For instance, if there is constant moving the authors suggest the use of a treadmill with varying speed to simulate real situations. As a consequence, conscious attention is required from the user. The second framework aims to evaluate divided attention, frequently distracting the user in order to simulate the use of a mobile system in a real world setting.

To evaluate both frameworks, experiences were conducted to assess their validity comparing them to real world evaluations. The frameworks were compared to an experience where users moved through a pedestrian street while using the mobile system. Overall, conclusions state that there are no significant differences between the use of the frameworks or real setting evaluations. In fact, the situation where most usability problems were identified was while the user was seating at a table.

However, these experiences reduced real settings and contextual influence to physical movement and degree of attention, introducing limitations on the evaluation. Using these techniques, it is extremely difficult to assess how users react to different social environments and the impact of public or privacy issues on the usability of the mobile system. Pering (Pering, 2006) conducted a study, which clearly concluded that users' behaviour changes according to the privacy settings of his/her location and working context and that these changes must be incorporated into applications and usability studies. This kind of issue is further noticeable when using location-enhanced technology that allows social communication.

Consolvo et al. state that it is crucial to understand why, when and what users want to share with each other and how this influences their social behaviour as well as the use of technology (Consolvo et al., 2005). Therefore, it is paramount to undertake evaluation studies with realistic settings (Duh et al., 2006; Nielsen et al., 2006), where more than physical characteristics of the environment are emulated. Following these findings, recent 
studies have addressed the need to evaluate mobile systems on actual scenarios and emerging techniques that aim to support these procedures (Duh et al., 2006; Hagen et al., 2005).

Hagen et al. categorized currently used techniques as mediated data collection, simulations and enactments and combinations. On the first, both users and the used technology collect usage data on natural settings. On the second, simulations of real settings are used whilst on the third, combinations of several techniques are utilised. These three categories comprise a set of different techniques which can rely on the use of specific data collecting hardware or on participants collecting data themselves. In fact, this last approach seems to be gaining momentum (Consolvo \& Walker, 2003; Froehlich et al., 2006).

The Experience Sampling Method (ESM) is a technique inherited from the field of psychology, which relies on questionnaires to gather usage information on natural settings. Participants of evaluation sessions, using mobile devices, are prompted with questionnaires, providing usability data that can be later analysed and statistically evaluated. Furthermore, since designers are not present during questionnaire filling-in, bias associated to observation is reduced. However, despite the positive results, this technique has some problems as well. It depends greatly on the participants' availability to respond to the questionnaires and to the content of each questionnaire. While user observation provides factual data and facilitates the detection of unexpected usability problems, questionnaires generally point and lead users at problem detection on specific directions. Nevertheless, the technique has proved to be useful. However, the practical advice on how to apply it is limited and suggestions on how to use it with complementary techniques, providing more trustful results is required.

Finally, some evaluation procedures that take advantage of specific hardware are also being experimented (Isomursi et al., 2004; Lukander, 2004; Thompson et al., 2004). In (Isomursi et al., 2004) the authors present an experience where users were filmed and followed while using a mobile device. However, given their presence and the public environment, the user experience was affected. Another alternative experimented on this work relied on users to capture images from other users while utilizing the applications. Moreover, since most of the photographs provided little information when analysed individually, some users also provided annotations of the events in which the photos were taken. However, once again users' annotations did not provide detailed information about usability issues.

Consequentially, the authors suggest a new approach called the "experience clip". The method is based on the usage of a camera phone and a PDA. The PDA contains the application that is being evaluated whereas the camera phone is used to capture clips that seem relevant to users. The experience demonstrated that the technique is able to provide rich data about user emotions and feelings. However, users have to be well motivated and have to be clearly instructed on what they are supposed to capture. Furthermore, the utilization of two devices generally requires the participation of more than one user. Similarly to what was discovered with the first experience that motivated the study, it is likely that the second presence and experience per se might change and influence the evaluation results. Nevertheless, the method provides better results than simulating real-life scenarios within laboratory settings. However, most of these require working software prototypes or expensive equipment.

Overall, on early stages of design, low-fidelity prototypes are scarcely used for mobile devices. Even so, there are some examples that proved to be successful (Svanaes \& Seland, 
2004). However, even these were tested in controlled scenarios with role playing within the laboratory (Duh et al., 2006; Kjeldskov \& Graham, 2003; Svanaes \& Seland, 2004). Furthermore, as previously demonstrated, it is important to conduct evaluations studies on actual settings since these highlight specific problems that might pass undetected on laboratory evaluations (Bodker \& Buur, 2002; Nielsen et al., 2006). Despite some existing techniques, currently used evaluation approaches are still ill suited to mobile scenarios and contexts of use. The existing literature, although providing some pointers and advice on how to conduct evaluation for mobile devices, generally does so for laboratory settings, contrasting with results that show the benefits and an imminent need for in-situ evaluation.

\section{A Design Methodology for Mobile Devices}

In order to overcome the stated difficulties, this chapter proposes a design methodology that focuses the new challenges introduced within design stages by mobility and the specific characteristics of mobile interaction. The methodology follows a user centred design approach and suggests the use of alternative techniques on all the initial design stages. As the experiences and examples that are provided here confirmed, the use of the methodology propelled the experimentation of new methods and tools that cope with the user centred design of mobile user interfaces, promoting in-situ design and evaluation and resulting in diverse solutions for various problems. The following sections detail how and which techniques can be used and some findings that emerged along the experiences that are presented.

\subsection{Pervasive Data Gathering}

For designers to understand currently faced problems and define more suited solutions, they must work together with future users and understand their needs so that created alternatives are innovative and usable.

As mentioned before, existing techniques provide designers with some directions to gather this information and analyze it. However, these techniques focus mainly on a specific setting and scenario. Even if they are repeated for every imagined scenario and setting, none of these methods provides means to relate the gathered requirements when settings are changed. Furthermore, the requirements and their causes are commonly lost during these transitions. This problem is generally non existent on fixed solutions since scenarios are usually immutable or usage restrictions, and their influence on the user's behaviour, are constant.

With mobile technology the case is hardly the same. Due to its pervasive and ubiquitous nature, the context, settings and usage scenarios are constantly changing, such as the requirements and needs that these imply. Therefore, it is crucial to understand the changes between settings and scenarios, which is possibly attainable with current methodologies, but it is also paramount to understand why and how these changes occurred. To achieve such results, the experiments that follow show how alternative techniques were used in order to gather information on pervasive activities on the field.

\subsubsection{Example 1}

On this experience, to tackle the aforementioned problems, alternative methods to evaluate and assess requirements on mobile settings were used. The envisioned application that was 
being designed aimed at supporting ubiquitous cognitive behavioural therapy, allowing patients to register their thoughts, emotions and problems throughout their daily lives on handheld devices (Sá \& Carriço, 2007). The tool also included relaxation procedures and tutorials accompanied by pro-active aids and hints that had to be displayed whenever the user showed (through his/her answers to short questions) signs of stress. Particular care was directed to understand how usage and user's behaviours were affected by the surrounding environment, emphasizing not only the changes but also their causes and effects. Firstly, a high level conceptual framework was established, pointing specific directions and highlighting crucial concerns that had to be taken into consideration (e.g., user position, activity and goal, environment settings - lighting or noise conditions, cooperation with others, social context).

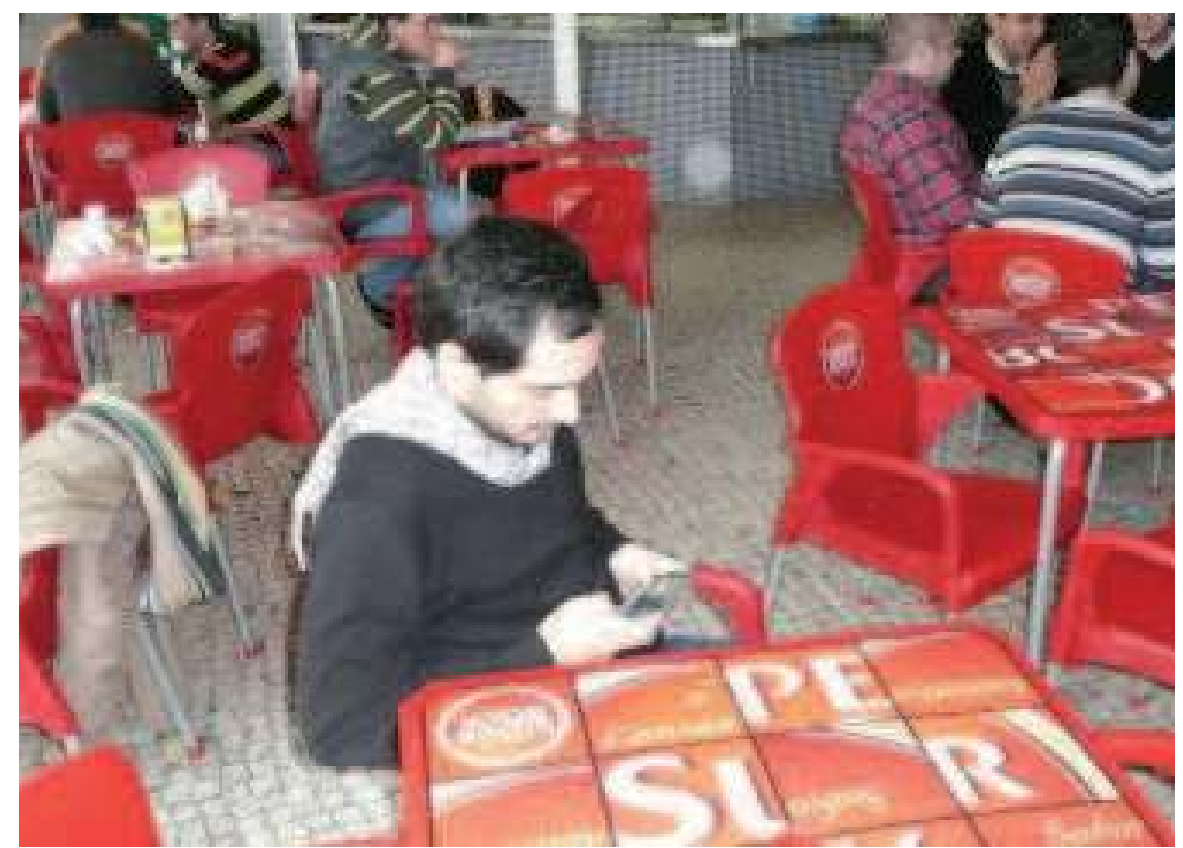

Fig. 1. User completing a task in-situ while seated at a coffee with some friends.

This framework was used to define and select some scenarios which included a set of details, derived from the framework, which needed to be taken into account while designing the envisioned applications, each including various contexts. The main goal was to assess the influence of personal, social, cultural, and cooperation issues on each context without disregarding dimensions that relate directly to the user and his/her behaviour. Accordingly, scenarios varied through multiple instances containing different user positions while working (e.g., walking, standing, seating, etc); different social/cultural events and distractions (e.g., conversation, school, work, meetings, on the bus), with special focus on those that could increase users' stress levels. This also permitted the understanding of user's reactions to transitions and subtle changes within contexts providing information on how the applications should adjust and correspond to these behaviours. 
To gather data in situations where users could not be observed or followed a specific type of questionnaires was distributed by users to be filled in-situ. Users were requested to complete the questionnaires while accomplishing their activities using their traditional means and procedures and to provide information on how, at that moment and on that location, a technological solution could be helpful. Moreover, these suggestions and answers had to be followed by a short description of the location and context in which the user responded to that questionnaire. Here, once again, details that were relevant were already included in the questionnaire through multiple-choice questions. This allowed designers to focus the details that mattered but still contextualising what users should provide.

The use of specific and contextual questionnaires and their completion in-situ provided insight on contextual details and information about the user's location even without the presence of the designer. Moreover, by requesting that information while the user was working or during a certain activity, the information about problems and suggestions of new features and solutions was much easier to recollect. Here, instead of using generic questionnaires, that could be filled through the internet or on any given time, this technique gathered answers and data in-situ, relying on users to characterise the environment in which they were, requesting information that designers needed but also stressing the details that seemed important to the user at that point.

\subsubsection{Example 2}

On the second example, the goal was to design a digital white cane for visual impaired users. Besides replacing the traditional cane, the digital one should include features that allowed users to get information of their context while moving around different locations. On this particular experience, the data gathering stage also followed different methods and procedures. Once again, a short amount of scenarios was established. Since these included most of the dimensions and concerns that were targeted by the framework used on the first experience, they covered a wide range of situations and variations. Locations included busy subway stations, corridors full of obstacles, supermarkets and open locations in order to assess users' needs and the way they used the traditional cane.

To gather data, the experience sampling method (Consolvo, 2007) and diary studies were used (Sohn et al., 2008). As previously explained, these methods rely on the users to gather data, requiring them to annotate and register situations where they feel that a solution could be used by describing their experience, the problems that they faced and, if possible, suggesting features and requesting functionalities that could aid them, much alike probing techniques (Hulkko et al., 2004).

To support this process without adding an extra burden to the visually impaired users, these registries were recorded on digital devices that users carried along with them (e.g., audio recorder, cell-phone with recording capabilities, PDA). This allowed users to record and register specific events, also providing a description of the settings and background of the experience in-situ, without the need to write or interrupt their current activity to do so. Moreover, since the recorders were continuously gathering data and the user's voice/thoughts, users were asked to keep providing information while walking. Overall, besides the requested information about the situation, the settings and encountered difficulties, this allowed designers to gather some information regarding transition between settings and on how users' behaviour and feelings changed while transiting from context to context. 


\subsubsection{Resulting Guidelines}

Overall, these two experiences showed that the data gathering process within mobile settings and during the design of mobile applications/tools is also achievable, with better results then when traditional techniques are used by using simple yet effective methods. Moreover, the used techniques relied on inexpensive material (e.g., paper and pencil) or even on material that was owned by the users (e.g., cell-phone to record audio). Since a large part of the data that was gathered did not require the designer's presence and direct observation, the entire procedure required less effort and posed fewer problems to users' privacy also providing less biased results. Also, it allowed data to be gathered by a larger number of users throughout the various iterations of each experience. Globally, from these two experiences, the following guidelines can be presented:

- The definition and selection of scenarios that address details that can affect the usability of the solution and the user's behaviour should be done and is paramount to the subsequent data gathering process.

- To gather data whenever direct observation is unachievable and when mobility and multiple settings are involved, contextual questionnaires can be provided to users. These should include questions that take into account the user's location, the environmental conditions, social context, etc.

- When completed in-situ, contextual questionnaires can provide information about the surrounding environment and facilitate recollection about problems and issues that emerge while performing specific activities.

- The experience sampling method, commonly applied to the evaluation of mobile applications, can also be used successfully to the initial data gathering stages.

- When necessary, data can be gathered with the aid of digital equipment (e.g., voice recorders, cell-phones) providing a less intrusive method. Moreover, since it is easier to talk than write, users generally provide richer data and more details. This approach also allows users to continue recording data while on-the-move, providing information even during transitions between contexts and settings.

\subsection{Early Stage Prototyping}

As already discussed, common low-fidelity prototyping techniques can result in misleading and erroneous results when used in mobile contexts to support the design of mobile applications. Accordingly, this section describes two of the experiences that took place during the design of several mobile systems and the lessons that emerged from that process. The prototypes that were used, how they were built and their advantages are shown, followed by a summary of the guidelines that can be applied on other projects and solutions.

\subsubsection{Example 1}

The main goal for the tool that was being conceived and designed during this experience was to allow students to use a mobile application to achieve a set of activities ubiquitously. The three main activities that needed to be included were the support for on-class test 
completion and annotation, homework completion and on-the-move analysis of content and registration of data. The initial stages followed the updated data gathering techniques that were addressed on the previous section. Once that data was analysed and design ideas started to be developed, the prototyping stage took place. While prototyping the application, and in order to provide users with a realistic experience and overview of the various design ideas, overcoming the problems mentioned in the related work, a frame was created using a light wooden material with about the same weight and size of a Palm Tungsten T3 (Figure 2).

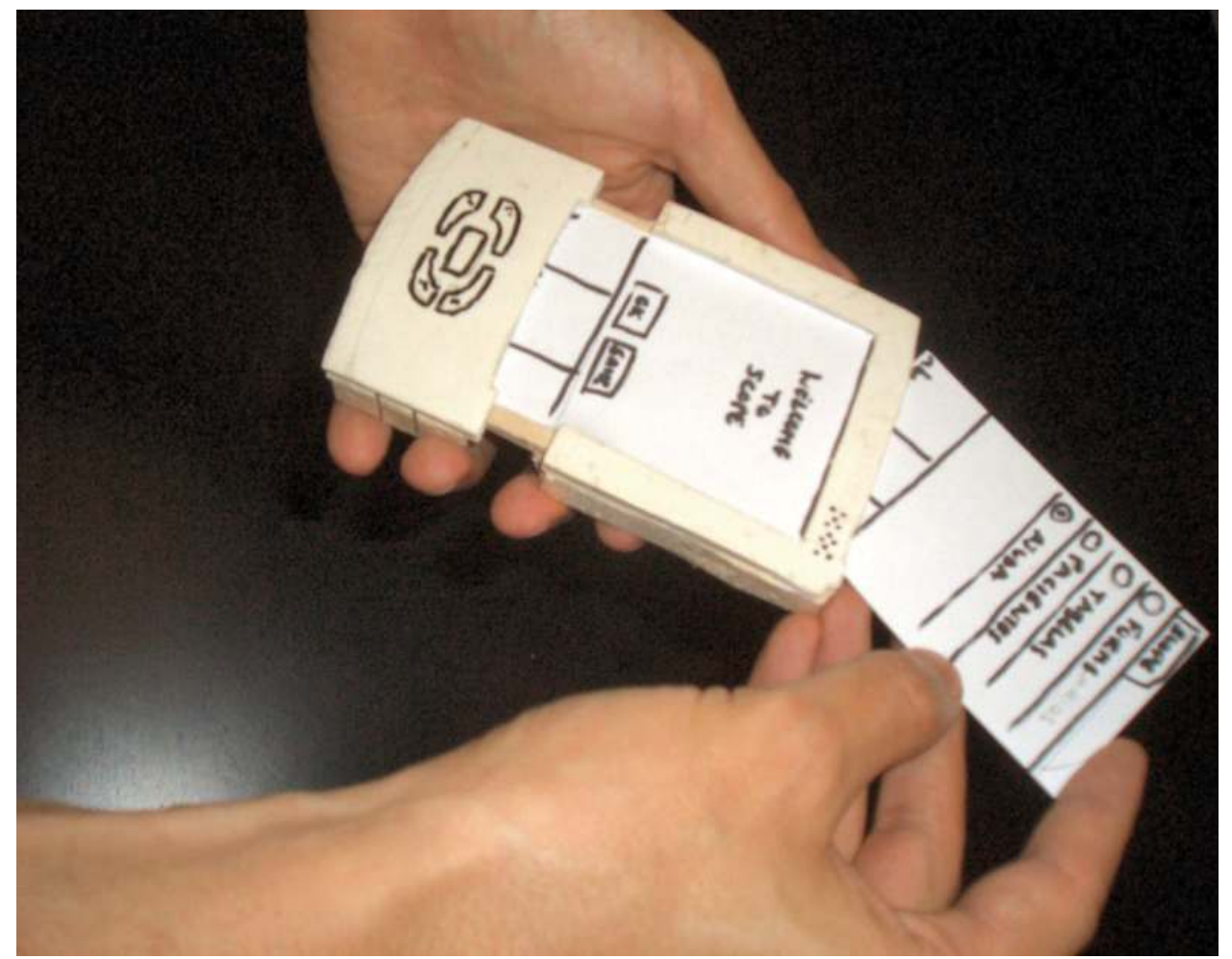

Fig. 2. Low-Fi prototyping frame mimicking a Palm T3 Tungsten. The slot allows users to easily exchange cards/sketches).

The frame had a small opening on the top, which allowed the sketched cards (screens) to be switched very easily. It took about forty minutes to build. Even so, it allowed users to have an accurate notion on the device and interaction techniques that they would use. By providing an actual solid artefact/prototype instead of only a set of cards, users were able to have a tangible experience, grasping details that would not have been visible with a traditional approach. Furthermore, it was quickly noticeable that this procedure and the resulting frame allowed the detection, and consequent correction, of a set of UI problems that would not have been found using just cards. For instance, buttons on the bottom of the screen had to be moved to the top for easier access and so that they would not move or 
disappear if the screen was extended (screen extension is a T3 feature). Also, buttons and lists that extended to, or were placed near, the borders of the screen were difficult to read and interact with.

This rigid frame and the realistic prototyping techniques also provided additional benefits. Using it, users were able to test the applications while walking from one place to another, switching screens at will. This showed that the size of some components that were used when the user was walking, which was a common situation in some tasks (e.g., taking notes or checking the agenda while walking on the schools corridors), needed to be larger because of the lower accuracy in this position. Overall, the use of the rigid frame allowed users to have a realistic idea of the design concept which, consequentially, provided designers with much richer information regarding their satisfaction towards the solution that was being developed.

\subsubsection{Example 2}

The second prototyping experience took place during the design of the digital white cane mentioned in the previous data gathering section. In this project, in order to show final users the design concept and to support in-situ evaluation, a low-fidelity prototype of the digital cane was built. The primary goal was to allow users to interact with it and to offer a handson experience that could provide user feedback regarding the envisioned tool as well as the software and functionalities that would be included on it. The prototype was created using paper, glue, a lighter's wheel to strike the spark (i.e., ferrocerium) and a strap. To support the audio warnings that were issued to the user a Bluetooth headset was also created.

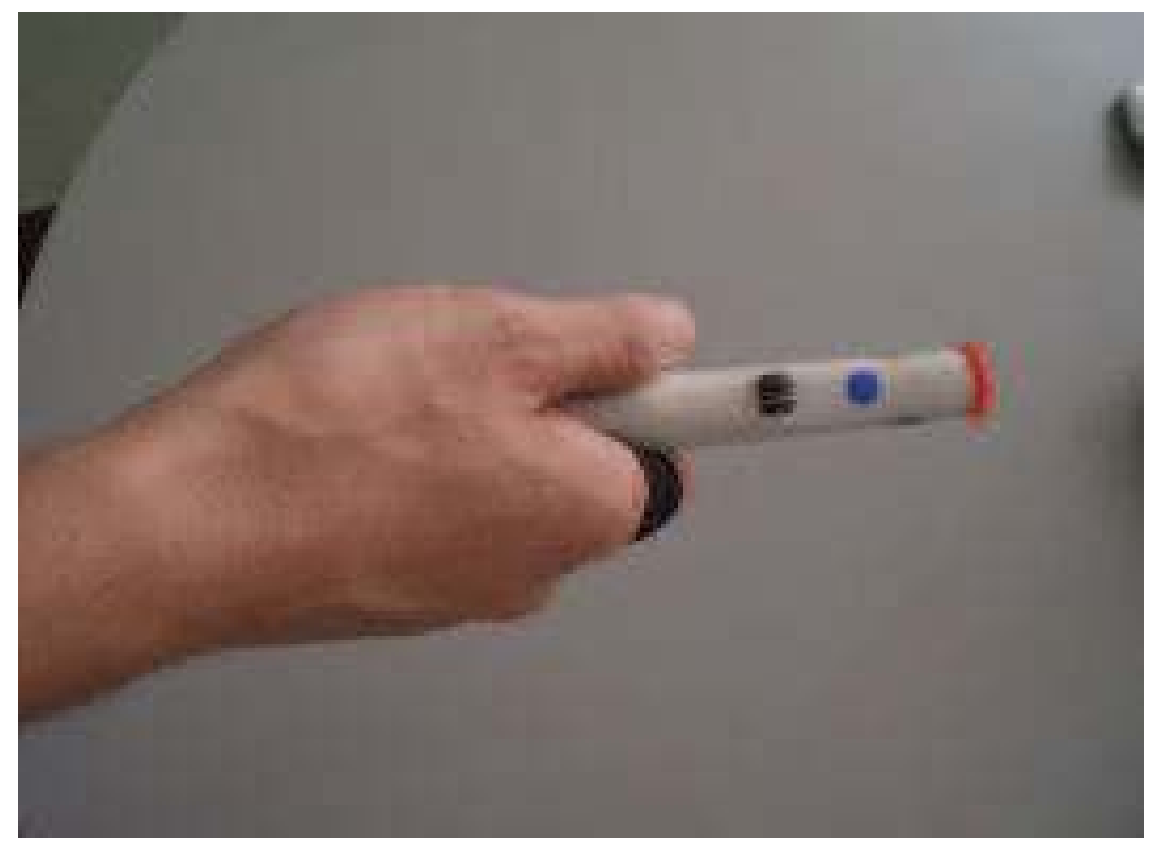

Fig. 3. Low-fi prototype of a digital white cane. The strap is adjustable and the tip includes an infra-red sensor that detects distance and reads bar-codes. The wheel on the centre allows users to select different options and to control the cane. 
The low-fi cane was then handed to several users that tried to re-enact their usual behaviour while walking within a furnished room and a corridor. Moreover, to test the prototype on realistic settings, users tried to use it on the same scenarios that were used for the data gathering stages. Accordingly, the prototype was used on a busy subway station, on a supermarket and several other locations.

The combined use of the rigid prototype and the realistic scenarios allowed designers and users to detect several problems that needed to be solved. The size of the cane was adjusted in order to easily fit in users' pockets. This was noticed while entering the subway, where users needed to secure themselves on the subway by grabbing the poles in order to maintain balance and needed to quickly store the cane. Moreover, it was noticed that using the cane on busy settings, where bumps occurred occasionally, the cane's strap needed to be adjustable so it would not fall. Moreover, new functionalities also emerged from these experiences on the field. For instance, when the prototype was used within a supermarket, one of the users suggested that besides being used as a proximity sensor, the cane should also use the infra-red sensor to read code-bars, providing information directly to the user's headset. Another innovation that emerged from this experience was the haptic feedback that was added to the cane. While walking within noisy locations or when talking with friends, users did not want to be disturbed by auditory warnings through the Bluetooth headset. Accordingly, the cane was updated in order to vibrate whenever a warning or new information was available.

\subsubsection{Resulting Guidelines}

The results from these projects and experiences suggested that these techniques can be used in various domains and situations. Accordingly, the following guidelines emerged:

- Common techniques and material do not apply directly for most mobile devices/applications.

- A careful distinction between the device prototypes and the application/UI prototype has to be made.

- The device prototype should be created using rigid materials in order to be used in real-life settings and it should have approximately the same dimensions of an actual device.

- When applicable, a slot where cards can be easily and quickly inserted and removed needs to be available. When this option is unavailable, alternatives that facilitate screen substitution should be provided.

- $\quad$ Sketches must be drawn with the same size of the device's screen using similar components and fonts to those available for a real device.

- The interaction type of the actual device should also be emulated (e.g. stylus/pen, joystick/small coloured drawing pin or a thumb tack).

- Using low-fidelity prototypes that resemble closely actual devices allows designers to test their design concepts with larger amounts of users still keeping the design costs low (e.g., handing 10 wooden prototypes to users, instead of real devices). Moreover, using this type of prototype brings the possibility for users to take the prototypes home and interact with them away 
from designers without the need to use their own devices (if they have any) or to purchase extra hardware.

- Although these hints might imply more effort from the designer, they are compensated with more accurate results.

\subsection{Mobile Evaluation}

Complementing the aforementioned procedures to create low-fidelity prototypes, the corresponding evaluation procedures must also be updated in order to take advantage of them. Particularly, it is essential to emphasize the importance of context and location while evaluating mobile applications. In fact, as already discussed, prototypes of various fidelities are usually tested on controlled settings and fictitious scenarios retracting from the evaluations sessions and preventing the detection of some usage errors.

\subsubsection{Example 1}

During the early evaluation of various applications, including the previously mentioned digital white cane, the context and settings of use proved to be of extreme importance. With the developed prototypes, users were able to carry the design concept with them to simulated and real situations. These findings led to the experimentation of similar evaluation sessions but using different prototypes. Accordingly, this procedure was also applied to the design of a GPS application that was being developed targeting mobile devices. The application was directed to users that needed to carry a mobile device along with them during their daily lives (e.g., traditional use of the GPS) with particular focus to highly physical activities (e.g., jogging, cycling, driving).

To test the GPS application users carried the sketches with simulated applications from one place to another, using them and exchanging them as needed during their activities. Besides evidencing some design problems that could occur, the ubiquitous evaluation sessions promoted participatory design, allowing users to engage actively on the process. In fact, most of the innovations appeared when testing the prototypes on real situations and scenarios. Since the frameworks were handed to users and they were free to use the prototypes whenever desired, most of the users involved in the process stated that whenever awkward design choices were found or whenever they thought there was a better solution, it was easier to immediately draw and annotate their idea. Here, the rigid frame that was used was also helpful, allowing users to easily write or draw on the cards or even create new ones without compromising the overall feeling of the prototype and experience.

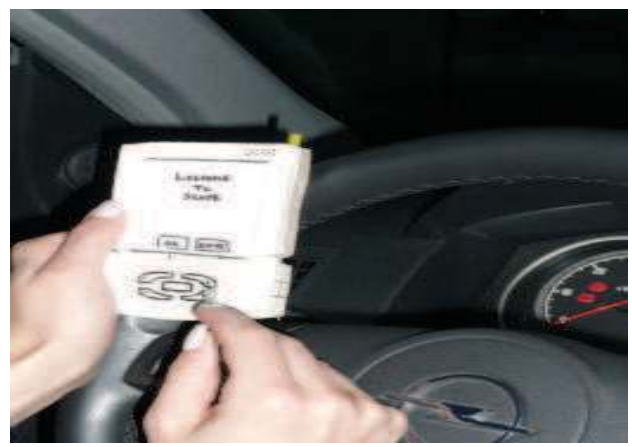




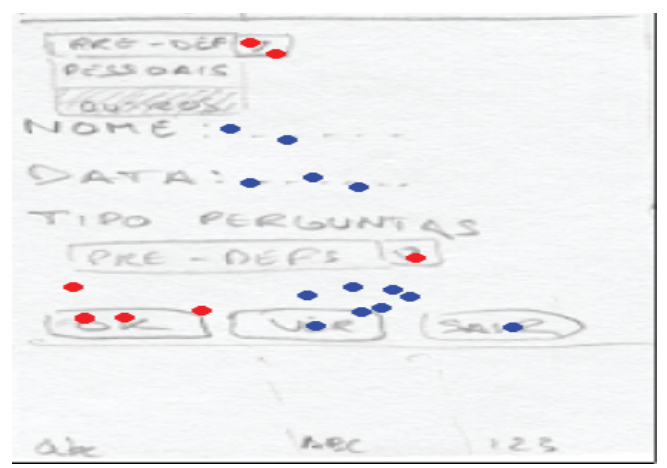

Fig. 4. Left: Low-fidelity prototype of a GPS application being used in-situ, while the user was in her car. Right: Sketch after an in-situ evaluation session.

To gather information regarding user activities while using the prototypes in-situ and to avoid following users around, users were also requested to use different colours for different tasks that they tried to accomplish while using the prototype (coloured pens were used in replacement of the usual stylus and/or fingers). The same procedure was suggested for different contexts and locations. By using specific colours for specific tasks or contexts, designers were able to detect problems that occurred in each of those contexts or activities. For instance, users were asked to use the red and blue colours for activities that were accomplished while walking. The sketches that were later returned to designers and that were marked with red paint clearly showed that users had lower accuracy towards buttons and that these needed to be enlarged (figure 4 on the right). Furthermore, users were asked to organise or "card-sort" the sketches when in specific contexts. This also allowed designers to notice that the same activities should have different sequences for different contexts, suggesting the need for a wider set of customization options on the application's user interface.

\subsubsection{Example 2}

On the second experience, to complement the evaluation information that was captured by the low-fidelity prototypes used according to the procedures that were introduced, a mobile video kit was used. The aim was to gather reliable information without requiring a large amount of evaluators following each user around while filming. The inexpensive video kit was comprised by a webcam that the user carried on his/her shoulder (figure 5). This camera weighed about $1 / 4$ pounds and was connected to a light ( 3 pounds) laptop computer that was kept in a backpack that the user also carried (figure 5). Overall, according to the users, the entire set of equipment was not very intrusive and required little effort to carry. The main concern regarding this kit was the embarrassment that it could cause while using it. The laptop had battery for 6 hours and could capture the same amount of video. The camera pointed at the average height to which users' held their device while using it. This position was established after several tries with different users on various positions/postures. It also followed the user's movement, providing information regarding the direction to which the user was moving and the objects that were in his/her way (figure 5). 
After the initial experiences, users were questioned about the procedure. The general opinion was that the kit was less intrusive than being followed by designer/evaluator during the day. Moreover, as most of the users stated, they were aware and uncomfortable with the kit on the initial minutes but got used to it very quickly and almost didn't notice it on the final stages of the evaluation sessions.

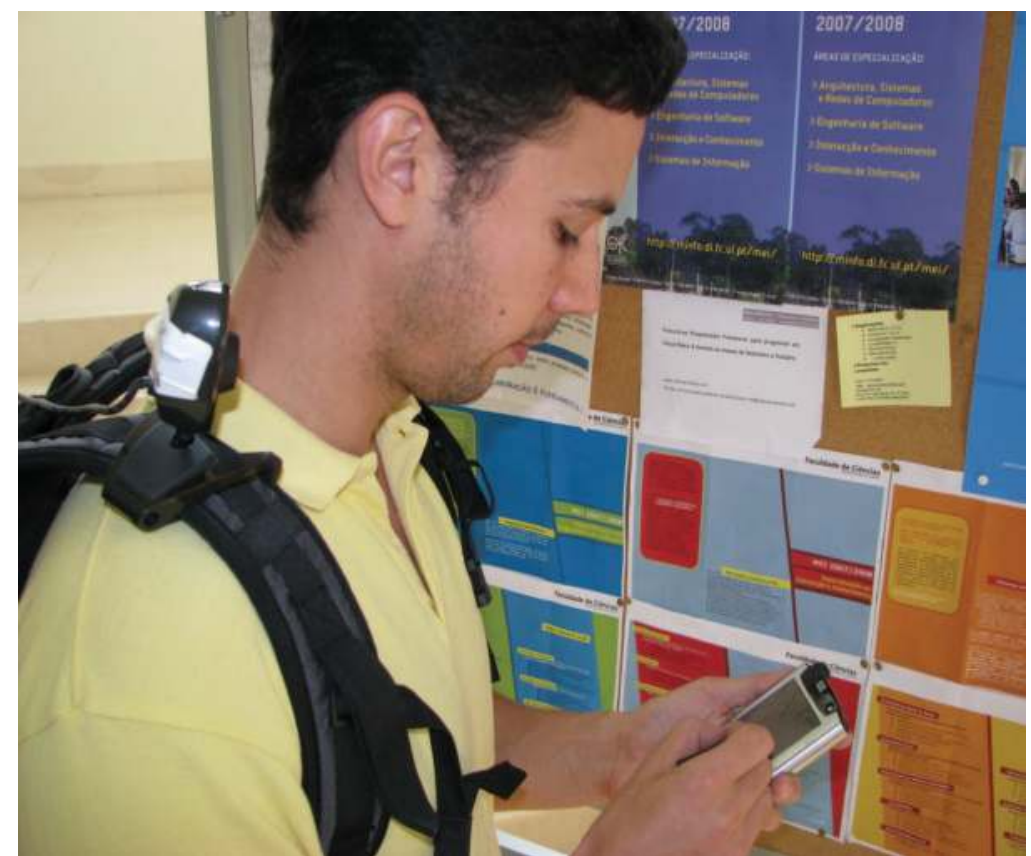

Fig. 5. Mobile video evaluation kit. The camera attached to the user's shoulder captures video footage of his interaction with the device/application.

In addition to the kit, and in order to gather unbiased qualitative data, collected when using the application while all the problems and flaws are easy to recollect, user satisfaction questionnaires were provided to the users once again. However, taking advantage of the video and audio capturing capabilities of the kit, users could express their opinions orally and even during interaction with the prototypes/devices (e.g., think-aloud). Moreover, since the context and environment in which the user was navigating was automatically captured by the kit, it was no longer necessary to request the user to provide this information.

The use of the kit allowed designers to detect other interaction details that were unnoticed when using only the previous techniques. For instance, the user's behaviour when interacting or cooperating with other users or even different postures and behaviours towards obstacles and paraphernalia that was used in concert with the prototypes were easily analysed by the designers and taken into account into the following prototypes. These positive results also propelled the use of the video kit even in later stages of evaluation with actual devices and software prototypes (figure 5). 


\subsubsection{Resulting Guidelines}

From the experiences that were conducted while evaluating several prototypes, even during later design stages, the following guidelines were encountered:

- Using rigid materials on the prototypes allows users to carry them, keep them in their pockets, take them home and participate on the sketches' design directly on the device, conducting evaluation on relevant locations.

- Context of use is essential for effective usability testing and, similarly to the initial data gathering stages, evaluation should be done in several possible scenarios that are sufficiently detailed to range a wide scope of settings and use contexts.

- The use of adequate material allows users to participate on the sketching process during contextual sessions, further promoting innovation and adequate ideas that cope with the contextual requirements.

- Tasks should be defined previously (e.g. using scripts), but the possibility of creating new features and test them on the spot should be provided.

- Colour schemes can be used to trace users' activities during and after the evaluation sessions. Moreover, if tasks/locations/screen arrangements are matched with specific colours or notations, evaluators can trace the users' behaviours without direct observation.

- Questionnaires can also be given to users together with the low-fidelity prototypes, so that they can complete them during out of the lab sessions. Here, the rigid prototyping frames also facilitate questionnaire filling. Moreover, using similar colour schemes to those previously mentioned, questionnaires can be filled with different colours according to specific locations or situations (e.g., around other users, at home, walking), eliciting problems that pertain to those using contexts.

- Mobile video capturing kits can be quickly arranged without using expensive material. Yet, these provide rich data and do not require designers to follow users constantly.

- The use of video and audio equipment also support a multi-media experience sampling method, relieving users from questionnaire answering and thought registering tasks. With the media hardware, users can simply think aloud about the current experience or video footage capturing the interaction and the surroundings can be used.

\section{Discussion and Further Research Directions}

So far, the work presented in this chapter has allowed the detection of several shortcomings on existing design methodologies when applied to the design of mobile applications and corresponding user interfaces. These findings, and the "on-the-fly" solutions that were used to cope with them, motivated the development of a methodology with techniques that apply specifically to mobile devices and ubiquitous applications. Globally, these problems led to the belief it was necessary to introduce solid guidelines on the various stages of design focusing on the mobility factor and on the users' varying context.

On the data gathering processes, the updated techniques provided means for designers to gather contextual information by requiring users to complete questionnaires in-situ. Moreover, the use of audio recording when using the ESM method permitted a pervasive 
data gathering process, allowing designers to understand users' behaviour while transiting between scenarios/settings. While not as rich or complete as user observation and contextual inquiry, these techniques are especially adequate to the initial stages of design because of their low-cost, ease of use and range. Moreover, they are particularly adequate to mobile scenarios since user observation on-the-move is difficult to achieve and inherently impossible to accomplish in every imaginable scenario/context. Also, it is noteworthy that these techniques do not aim at replacing traditional ones but aim at complementing them.

Regarding the prototyping stage, as the results showed, the used techniques gave users a much more detailed idea of the designers' vision and facilitated their in-situ evaluation. In general, although at times these procedures might require added effort to apply, they enhance the evaluation process and the detection of errors in very early stages. In fact, these techniques highlighted the close relationship between the prototyping and the evaluation stage since procedures often merge and have a direct impact and influence between these two design stages.

For the evaluation stage, the experiences that were undertaken clearly demonstrated that insitu trials and evaluation stages can be successfully achieved even without the use of expensive material. By using adequate prototyping techniques and evaluation methods, designers can easily test their design concepts and ideas on realistic settings. Moreover, some of these techniques (especially the use of the video kit) can also be used on the initial data gathering stages.

Finally, it is noteworthy to mention that the initial setup and definition of scenarios, each containing several aspects and dimensions that are paramount to mobile activities, which can be contextualized to particular projects, is highly beneficial and influences the effectiveness of the entire design process.

As a result from the advances and concepts that emerged from these experiences, a prototyping framework for mobile devices, which takes advantage of these guidelines, is being developed. Overall, the tool aims at supporting prototyping and evaluation of applications with the peculiarity of creating the prototypes on actual devices. Some of its contributions are (1) the support for prototypes with various fidelities; (2) an automatic mechanism to gather contextual data; (3) the inclusion of ESM and probing techniques; (4) the support of on-the-spot participatory design and (5) the use of pre-established design guidelines. Accordingly, the tool will support design through the in-situ generation and evaluation of prototypes, using actual devices, even at very early design stages.

\section{Conclusions}

Current design approaches, although providing extensive detail on various stages of the design process, fail to support most of the related activities and tasks when mobility of the system is paramount. Existing techniques, particularly for initial stages of development and evaluation, are rarely applied, especially taking into account the volatile settings in which the applications are likely to be used. Furthermore, these techniques aren't always applicable and, when ill-used, hinder the overall process.

In this chapter, the problems that emerged while using common user centred design methodologies on the development of a set of ubiquitous systems were described. Throughout these experiences, mobility, pervasiveness and ubiquity required the adoption of new techniques and the adjustment of existing ones in order to cope with the challenges 
that derived from the constant change of context and multitude of settings that could be envisioned for each specific system.

Overall, these techniques and resulting findings allowed the gathering of data on relevant locations and settings on a pervasive manner. Moreover, they propelled the detection of user interface problems on both low-fidelity and high-fidelity prototypes during the various evaluations sessions that were conducted. The use of methods that connect the various stages and that were conceived in order to facilitate the transition and evolution from stage to stage enhanced the overall process, resulting in sounder solutions and usable products and application. In comparison with existing techniques, which were used on initial stages of these processes, the new techniques provided insight to problems which would not have been detected otherwise.

This chapter provided an overview on existing problems with mobile interaction design, particularly addressing the early design stages, and proposed techniques and solutions that can be applied to projects in various domains. As a validation and as a medium to clearly show how to apply each technique, several experiences were presented and discussed highlighting the methods that had positive outcomes and the procedures that were used.

\section{Acknowledgements}

This work was supported by LaSIGE and FCT, through project JoinTS, through the Multiannual Funding Programme and scholarship SFRH/BD/28165/2006.

\section{References}

Barnard, et al. Capturing the effects of context on human performance in mobile computing systems. Personal and Ubiquitous Computing, Vol. 11, No.46, pp.81-96, 2005.

Black, J.T. \& Hawkes, L.W. (2006). A prototype interface for collaborative mobile learning. In IWCMC'06, 1277-1282, ACM Press, Vancouver, British Columbia, Canada.

Beyer, H., Holtzblatt, K., 1998. Contextual Design: Customer Centered Approach to Systems Design, Academic Press, San Francisco, CA, USA.

Bodker, S. \& Buur, J. (2002). The design collaboratorium - a place for usability design. Transactions on Computer Human-Interaction, 9, 152-169.

Brewster, S. (2002). Overcoming the lack of screen space on mobile computers. Personal and Ubiquitous Computing, Vol. 6, No. 3, 188-205.

Carter, S., et al. Momento: Support for Situated Ubicomp Experimentation. CHI'07, pp. 125134, ACM.

Coble, J.M., Karat, J. \& Kahn, M.G. (1997). Maintaining a focus on user requirements throughout the development of clinical workstation software. In Proceedings of the SIGCHI conference on Human factors in computing systems CHI'97.

Chandler, C.D., Lo, G. \& Sinha, A.K. (2002). Multimodal theater: Extending low fidelity paper prototyping to multimodal applications. In CHI2002

Extended Abstracts, CHI 2002, ACM, Minneapolis, Minnesota,USA.

Connelly, K.H., Rogers, Y., Siek, K.A., Jones, J., Kraus, M.A., Perkins, S.M., Trevino, L.L. \& Welch, J.L. (2005). Designing a pda interface 
for dialysis patients to monitor diet in their everyday life. In Proceedings of the 11th Human Computer Interaction International Conference, HCII05, Las Vegas, USA.

Consolvo, S. and M. Walker, Using the Experience Sampling Method to Evaluate Ubicomp Applications. IEEE Pervasive Computing, 2003. 2(2): p. 24-31.

Consolvo, S., Roessler, P., Shelton, B., LaMarca, A. \& Schilit, B. (2004). Technology for care networks for elders. IEEE Pervasive Computing,3, 22-29.

Consolvo, S., Smith, I.E., Mathews, T., LaMarca, A., Tabert, J. \&

Powledge, P. (2005). Location disclosure to social relations: Why, when and what people want to share. In CHI $2005,81-90$, ACM, Portland, Oregon, USA.

Duh, H.B.-L., G.C.B. Tan, and V.H.-h. Chen. Usability Evaluation for Mobile Device: A Comparison of Laboratory and Field Tests. in Mobile HCI'06. 2006., Finland, ACM.

Frishberg, L. (2006). Presumptive design, or cutting the looking-glass cake. Interactions, 8, 18-20.

Froehlich, J., Chen, M., Smith, I. \& Potter, F. (2006). Voting with your feet: An investigative study of the relationship between place visit behaviour and preference. In UbiComp 2006 , Orange County, California, USA.

Froehlich, J., et al. MyExperience: A System for In Situ Tracing and Capturing of User Feedback on Mobile Phones. MobiSys'07, pp. 57-70, ACM.

Hagen, P., et al. Emerging Research Methods for Understanding Mobile Technology Use. Proc. of OZCHI 2005, Camberra, Australia, 2005.

Hanington, B.M. (2006). Interface in form: Paper and product prototyping For feedback and fun. Interactions, 8, 28-30.

Holmquist, L.E. (2005). Generating ideas or cargo cult designs? Interactions, $7,48-54$.

Holtzblatt, K., Wendell, J.B. \& Wood, S. (2005). Rapid Contextual Design: A How-To Guide to Key Techniques for User-Centered Design. Morgan Kaufmann, San Francisco, CA, USA.

Hulkko, S., et al. Mobile Probes. NordiCHI'04. ACM.

Isomursi, M., Kuutti, K. \& Vainamo, S. (2004). Experience clip: Method for user participation and evaluation of mobile concepts. In Proceedings of Participatory Design Conference 2004, ACM, Toronto, Canada.

Jan, Blom et al. Contextual and Cultural Challenges for User Mobility Research, Communications of the ACM, Vol 48, No.7, 37-41, 2005.

Jones, M. and G. Marsden, Mobile Interaction Design. 2006, West Sussex, England: John Wiley \& Sons Ltd.

Kangas, E. \& Kinnunen, T. (2005). Applying user-centered design to mobile application development. Communications of the ACM, 48, 55-59.

Kelley, J. (1984) An iterative design methodology for user-friendly natural language office information applications. Trans. Office Information Systems, 2.

Kjeldskov, J. and C. Graham, A Review of Mobile HCI Research Methods. Mobile HCI'03. Vol. 2795/2003. 2003: Springer Berlin / Heidelberg. 317-335.

Kjeldskov, J. and J. Stage, New Techniques for Usability Evaluation of Mobile Systems. International Journal of Human Computer Studies, Elsevier, 2003.

Lee, Young E. Interface Design for Mobile Commerce. Communications of the ACM,Vo.46, No.12, 2003, 49-52. 
Lukander, K. (2004). Measuring gaze point on handheld mobile devices. In Extended Abstracts of CHI2004, ACM, Vienna, Austria.

Marcus, A., Chen, E., Designing the PDA of the Future. Interactions, 9:1, pp.32-44, ACM, 2002.

Mayhew, D.J. (1999). The Usability Engineering Lifecycle. Morgan Kaufmann, San Francisco, CA, USA.

Mazzone, E., Horton, M. \& Read, J. (2004). Requirements for a multimedia museum environment. In Proceedings of the third Nordic conference on Human computer interaction NordiCHI '04.

Nakajima, T., How to Reuse Existing Interactive Applications in Ubiquitous Computing Environments? SAC'06, Dijon, France, pp 1127-1133.

Nielsen, C.M., et al. It's Worth the Hassle! The Added Value of Evaluating the Usability of Mobile Systems in the Field. NordiCHI'06. ACM.

Pering, T. (2006). Evaluating the privacy of using mobile devices to interact in public places. In Permid 2006, Workshop at Pervasive 2006, Dublin, Ireland.

Raptis, D., N. Tselios, and N. Avouris. Context-based Design of Mobile Applications for Museums: A Survey of Existing Practices. in MobileHCI'05. ACM.

Reich P., et al, The LiLiPUT Prototype. CHI'07 (extended abstracts),pp.1833-1838,ACM.

Tiago Reis, Marco Sá, Luís Carriço. Designing Mobile Multimodal Artefacts. Proceedings of ICEIS 2008, the 10th International Conference on Enterprise Information Systems, Barcelona, Spain, INSTICC, June 2008.

Robert A. Virzi, D.K., Jeffrey L. Sokolov (1996). Usability problem identification using both low- and high-fidelity prototypes. In Proceedings of $\mathrm{CHI}$ 1996, ACM, Vancouver, Canada.

Rosenberg, D. (2006). Revisiting tangible speculation: 20 years of ui prototyping. Interactions, 8, 31-32.

Sá, M., Carriço, L., Low-Fi Prototyping for Mobile Devices. In Proceedings of CHI'06 (extended abstracts), Montréal, Canada, pp. 694-699, ACM.

Sá, M., Carriço, L., Handheld Devices for cooperative Educational Activities. In Proc. of SAC'06, Dijon, France, pp. 1145-1149, ACM Press, April, 2006

Sá, M. Carriço, L., Antunes P., Ubiquitous Psychotherapy. IEEE Pervasive Computing, vol. 6, n⿳0 1, pp. 20-27, IEEE Press, 2007.

Sá, M. Carriço, L., Lessons from Early Stages Design of Mobile Applications. Proceeding of MobileHCI'08, 10th International ACM Conference on Human Computer Interaction with Mobile Devices and Services, Amsterdam, Netherlands, 2008.

Scholtz, J. \& Consolvo, S. (2004). Toward a framework for evaluating ubiquitous computing applications. IEEE Pervasive Computing, 2, 82-86.

Svanaes, D. and G. Seland. Putting the users center stage: role playing and low-fi prototyping enable end users to design mobile systems. in CHI'04, Vienna, Austria ACM.

Sohn, T., Li, K. A., Griswold, W. G., and Hollan, J. D. 2008. A diary study of mobile information needs. In Proceeding of the Twenty-Sixth Annual SIGCHI Conference on Human Factors in Computing Systems (Florence, Italy, April 05 - 10, 2008). CHI '08. ACM.

Thompson, K.E., Rozanski, E.P. \& Haake, A.R. (2004). Here, there, anywhere: Remote usability testing that works. In Proceedings of SIGITE'04, 
ACM, Salt Lake City, Utah, USA.

Weiss,S.(2002).Handheld Usability.Wiley \& Sons, England. 


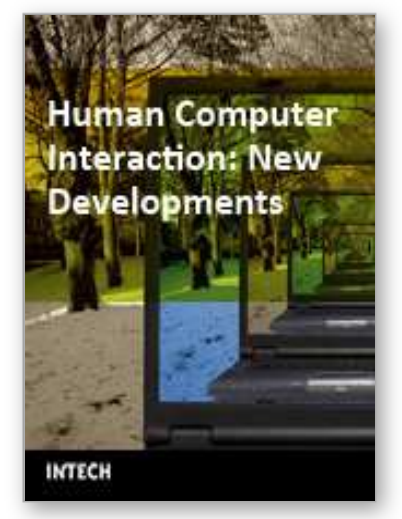

\author{
Human Computer Interaction: New Developments \\ Edited by Kikuo Asai
}

ISBN 978-953-7619-14-5

Hard cover, 382 pages

Publisher InTech

Published online 01, October, 2008

Published in print edition October, 2008

The book consists of 20 chapters, each addressing a certain aspect of human-computer interaction. Each chapter gives the reader background information on a subject and proposes an original solution. This should serve as a valuable tool for professionals in this interdisciplinary field. Hopefully, readers will contribute their own discoveries and improvements, innovative ideas and concepts, as well as novel applications and business models related to the field of human-computer interaction. It is our wish that the reader consider not only what our authors have written and the experimentation they have described, but also the examples they have set.

\title{
How to reference
}

In order to correctly reference this scholarly work, feel free to copy and paste the following:

Marco de Sa, Luis Carrico and Carlos Duarte (2008). Mobile Interaction Design: Techniques for Early Stage In-Situ Design, Human Computer Interaction: New Developments, Kikuo Asai (Ed.), ISBN: 978-953-7619-14-5, InTech, Available from:

http://www.intechopen.com/books/human_computer_interaction_new_developments/mobile_interaction_desig n_techniques_for_early_stage_in-situ_design

\section{INTECH}

open science | open minds

\section{InTech Europe}

University Campus STeP Ri

Slavka Krautzeka 83/A

51000 Rijeka, Croatia

Phone: +385 (51) 770447

Fax: +385 (51) 686166

www.intechopen.com

\section{InTech China}

Unit 405, Office Block, Hotel Equatorial Shanghai

No.65, Yan An Road (West), Shanghai, 200040, China 中国上海市延安西路65号上海国际贵都大饭店办公楼 405 单元

Phone: +86-21-62489820

Fax: +86-21-62489821 
(C) 2008 The Author(s). Licensee IntechOpen. This chapter is distributed under the terms of the Creative Commons Attribution-NonCommercialShareAlike-3.0 License, which permits use, distribution and reproduction for non-commercial purposes, provided the original is properly cited and derivative works building on this content are distributed under the same license. 